\title{
Use of the BICAMS in Patients with Multiple Sclerosis During Attack Period: A Prospective Controlled Study
}

\author{
(1) Ozan Ozturk1, (1) Bilge Piri Cinar22, (1) Serkan Ozakbas'1, \\ ${ }^{1}$ Dokuz Eylul University Faculty of Medicine, Department of Neurology, Izmir, Turkey \\ 2Zonguldak Bulent Ecevit University Faculty of Medicine, Department of Neurology, Zonguldak, Turkey
}

\section{Abstract}

Objective: Assessment of cognitive impairment, which cannot be evaluated with ordinary scales, in multiple sclerosis (MS) has been thought to be beneficial. MS significantly impairs the quality of life. During an MS attack, using a tool such as the brief international cognitive assessment for MS (BICAMS) battery, which can be administered quickly and applied and interpreted by individuals without neuropsychology training, provides a rapid and broad assessment of the patient's status.

Materials and Methods: The symbol digit modalities test (SDMT), california verbal learning test II (CVLT II), brief visuospatial memory test-revised (BVMT-R), and expanded disability status score were applied before treatment, at the end of treatment, and at the first month after treatment to evaluate mental and cognitive status in the acute period in patients with MS, who met the inclusion criteria.

Results: This study enrolled 49 patients with MS (F/M, 30/19) and 25 healthy controls (F/M, 16/9). The CVLT II scores were significantly higher after treatment than at baseline evaluation $(p<0.0001)$. However, no significant difference was observed in data obtained at the end of treatment and at the first month after treatment $(p=0.517)$. The CVLT II scores before treatment and at the first month increased in both groups, which was significantly higher in the MS group than in the control group ( $p=0.002$ ). In the MS group, a marked increase was found in the number of correctly drawn figures on the BVMT-R at the end of treatment compared with that drawn before treatment $(p<0.0001)$. Values obtained at the first month after treatment were significantly higher than those measured before treatment $(p<0.0001)$. In the MS group, the mean numbers of symbols drawn correctly in $90 \mathrm{~s}$ on the SDMT were $42.04 \pm 11.73$ on the first visit and $45.40 \pm 16.32$ at the end of treatment, indicating a significant improvement $(p=0.003)$, and the mean number of symbols drawn correctly at the first month was $48.14 \pm 15.03$.

Conclusion: This study found that the BICAMS battery was effectively used in the acute period, the short total application period was 15 min, and a neuropsychologist was not required for application and interpretation. These results suggest the potential application of BICAMS battery in daily practice.

Keywords: Multiple sclerosis, attack, cognitive impairment, cognitive tests

\section{Introduction}

Multiple sclerosis (MS) is a chronic, inflammatory, demyelinating disease of the central nervous system. The disease course may be marked by successive periods of worsening and improvement or by the gradual progression of neurological findings. Manifestations that last at least $24 \mathrm{~h}$, with at least 1 month between these and another episode, and producing one or more clinical findings are defined as attacks. Intravenous methylprednisolone (IVMP) is widely used in the treatment of MS attacks. Cognitive impairment is a manifestation seen in all stages from MS onset and in all subtypes, independent of physical disability, and compromises the therapeutic process and quality of life.

Cognitive impairment, which is frequently seen in patients with MS, that adversely affected the therapeutic process and quality of life of the patient has recently attracted considerable interest and has become the main focus of several studies. Rates of cognitive impairment in MS are as high as 43\%-70\% (1), and cognitive impairment is seen in all MS subtypes regardless of physical disability. Cognitive impairment associated with

Address for Correspondence: Bilge Piri Cinar, Zonguldak Bulent Ecevit University Faculty of Medicine, Department of Neurology, Zonguldak, Turkey E-mail: bilge.cinarpiri@gmail.com.tr ORCID-ID: orcid.org/0000-0002-4884-0717

Received: 23.06.2021 Accepted: 01.08.2021

${ }^{\circ}$ Copyright 2021 by the Journal of Multiple Sclerosis Research published by Galenos Publishing House. 
the duration and course of MS and the patient's education level can be observed at all stages from disease onset $(2,3)$. Cognitive functions that are particularly affected in patients with MS include the effectiveness and speed of information processing, executive functions, and long-term memory. Simple attention and verbal skills tend to be generally preserved in the subsequent stages of the disease.

The expanded disability status scale (EDSS) is frequently employed to determine disability levels in MS, but it is not sufficient for the assessment of cognitive status. Comprehensive neuropsychological evaluations, such as the brief repeatable battery neuropsychological tests and minimal assessment of cognitive function in multiple sclerosis, are useful in the assessment of cognitive impairment (4). However, these have limitations, such as being time-consuming and requiring experienced neuropsychologists for interpretation. Thus, in recent years, there has been a particular focus on practical alternatives for determining cognitive impairment in patients with MS, resulting in the development of the brief international cognitive assessment for MS (BICAMS) battery. The BICAMS battery consists of the symbol digit modalities test (SDMT), california verbal learning test II (CVLT II), and brief visuospatial memory test-revised. The battery possesses significant advantages, such as short application time (mean 15-20 min) and administration and interpretation by individuals without neuropsychological training (5-9).

The primary aim of this study was to investigate the presence of cognitive impairment during MS attack using the BICAMS battery. The secondary aim was to evaluate the feasibility of the BICAMS battery in evaluating response to treatment in the attack period of MS based on early and late period cognitive functions.

\section{Materials and Methods}

\section{Patient Enrolment}

This study included patients diagnosed with MS according to McDonald 2017 criteria(9), diagnosed with relapse, and planned for IVMP treatment. Patients were informed that evaluations would be conducted before attack treatment, at the end of treatment, and 1 month after treatment. Written consent was obtained from patients agreeing to participate in the study. Healthy volunteers who met the inclusion criteria were enrolled as the control group. In the MS group, patients aged 18-55 years who had a definite clinical diagnosis of MS [relapsing-remitting MS (RRMS) or secondary progressive MS (SPMS)], who was in the attack period, who had no more than 2 weeks of relapse since the onset of the current attack, who had sufficient cognitive ability to provide information about histories, and who had signed informed consent forms were included. Individuals with sufficient cognitive levels for providing information about their histories and who had signed informed consent forms were enrolled as the control group. Patients who used drugs that can affect clinical evaluation (antipsychotic use or continuous corticosteroid use including the previous 1-month period), who had neurological diseases that can affect clinical assessment, who had any other neurological and/or psychiatric disease that can affect the results of the cognitive tests were excluded. Ethics approval was obtained from the local ethics committee. All procedures performed in studies involving human participants were in accordance with the ethical standards of the institutional and/or national research committee and with the 1964 Helsinki Declaration and its later amendments or comparable ethical standards.

\section{Procedure}

In the MS group, neurological examinations with the EDSS were performed before treatment, end of treatment, and after 1 month of treatment, including the SDMT, CVLT II, and BVMT-R tests. In the control group, the SDMT, CVLT II, and BVMT-R tests were applied at baseline and after 2 weeks. Patients in the study group were followed up for 1 month. The tests were administered to the patients before IP therapy, at the end of treatment (on day 5, 7, or 10, depending on the response to treatment), and after 1 month. Tests were performed before IVMP therapy and at 14:00 hours at the latest so that fatigue developing over the day will not affect the cognitive tests.

\section{Statistical Analysis}

Statistical analyses were performed on SPSS 16.0 software for Windows. In addition to descriptive statistical methods (mean, standard deviation, and frequency), the chi-square test was used to compare rates in categorical variables, and the $p$ value calculated for the Fisher test was adopted where necessary. The non-parametric Mann-Whitney $U$ test was applied in the comparison of two non-normally distributed independent variables and Wilcoxon's test was performed for the means of two dependent groups. Spearman's correlation analysis was applied to determine the direction and level of correlation between variables, and $\mathrm{p}$ values $<0.05$ were regarded as significant for all results.

\section{Results}

This study included 49 patients with MS (F/M, 30/19) and 25 healthy controls (F/M, 16/9). No significant difference was observed between the two groups in terms of gender or education levels ( $p=0.51$ and $p=0.07$, respectively). The median age of the MS group was 37 [mean age, $36.41 \pm 8.37$ (21-51)] years. The median age of the control group was 38 [mean age, $37.40 \pm 9.58$ (20-55)] years, and no significant differences were observed between the groups ( $p=0.50)$. In the MS group, the mean EDSS scores were $3.77 \pm 1.35$ (2-6.5) before treatment, $2.57 \pm 1.21$ (1-5) at the end of treatment, and 2.12 \pm 1.20 (04.5) at the first month after treatment. The EDSS scores at the first month were significantly lower than the pre- and post- 
treatment values $(p<0.0001$ and $p<0.0001$, respectively) Significant improvement was also noted in the EDSS scores after treatment compared with those before treatment $(p<0.0001)$.

Regarding cognitive functions, verbal memory in the MS group was assessed using the CVLT. Accordingly, the mean numbers of words recalled correctly were $49.90 \pm 11.46$ before treatment, $56.84 \pm 11.91$ after treatment, and $57.69 \pm 10.79$ at the first month after treatment. No significant difference was determined between pre- and post-treatment values, but the difference between post-treatment and first-month values was significant $(p<0.0001$ and $p=0.517$, respectively). In the control group, the mean numbers of words recalled correctly were $53.96 \pm 9.4$ at the first visit and $55.96 \pm 9.67$ at the first month, showing a significant difference $(p<0.0001)$. No significant difference was observed when the MS group was compared with the control group at either the first visit or the first month ( $p=0.17$ and $p=0.337$, respectively) (Table 1 ). Comparison of the change in first-month CVLT scores compared with pretreatment values revealed an increase in both groups, but this increase was significantly higher in the MS group than in the control group $(p=0.002)$.

The number of correctly drawn figures on the BVMT-R was calculated at all three time points. In the MS group, the mean numbers of correctly drawn figures were 20.59 \pm 8.41 before

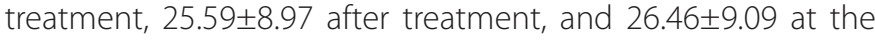
first month after treatment. A significant increase in numbers of correctly drawn figures was observed after treatment compared with that before treatment $(p<0.0001)$. First-month values were significantly higher than pretreatment values $(p<0.0001)$, but no significant difference was found when compared with post-treatment numbers $(p=0.10)$. In the control group, the mean numbers of correctly drawn figures in the first visit were 26.80 \pm 3.55 , and the difference between the MS and control groups was significant ( $p=0.001)$. Moreover, in the control group, the mean numbers of correctly drawn figures at the first month was 27.96 \pm 2.92 , and no significant difference was determined between the MS and control groups in terms of the numbers of correctly drawn figures on the BVMT-R at all three time points $(p=0.50)$ (Table 2). Comparison of the mean numbers of correctly drawn figures revealed a significant difference between the first visit and end of the first month on all three

\begin{tabular}{|c|c|c|c|}
\hline & $\begin{array}{l}\text { MS group } \\
\text { (mean } \pm \text { SD) }\end{array}$ & $\begin{array}{l}\text { Control group } \\
\text { (mean } \pm \mathrm{SD} \text { ) }\end{array}$ & $\mathbf{p}$ \\
\hline Pretreatment & $49.90 \pm 11.46$ & $53.96 \pm 9.4$ & 0.17 \\
\hline Post-treatment & $56.84 \pm 11.91$ & - & - \\
\hline First month & $57.69 \pm 10.79$ & $55.96 \pm 9.67$ & 0.337 \\
\hline
\end{tabular}

CVLT: California verbal learning test, MS: Multiple sclerosis, SD: Standard deviation
BVMT-R trials $(\mathrm{p}=0.001)$. Comparison of changes in pretreatment and first-month BVMT-R scores revealed an increase in scores in both groups, but the change was significantly greater in the MS group than in the control group $(p=0.005)$ (Figure 1).

In the MS group, the mean numbers of figures drawn correctly within $90 \mathrm{~s}$ on the SDMT were $42.04 \pm 11.73$ at the first pretreatment visit and 45.40 \pm 16.32 after treatment, indicating significant improvement compared with the pretreatment period ( $p=0.003$ ). Moreover, the mean number of correctly drawn figures on the first month was 48.14 15.03 , which was significantly higher than both the pretreatment $(p<0.0001)$ and post-treatment ( $p=0.005)$ values. In the control group, the mean number of correctly drawn figures on the SDMT within $90 \mathrm{~s}$ at the first visit was $47.56 \pm 8.76$, which was not significantly different from the value in the MS group ( $p=0.055)$. In the control group, the number of correctly drawn figures at the first month was 49.24 \pm 9.06 , which was also not significantly different from the first visit value $(p=0.08)$ (Table 3). Moreover, the mean numbers of correctly drawn figures at the first visit and the first month were not significantly different $(p=0.001)$ (Figure 2). Comparison of changes in pretreatment and firstmonth SDMT scores revealed an increase in both groups, but the increase was significantly greater in the MS group than in the control group $(p=0.001)$.

Degrees of correlation between EDSS scores and results of cognitive tests applied were then examined. A weak correlation was found between CVLT II and EDSS before treatment, at the end

\begin{tabular}{|l|l|l|l|}
\hline \multicolumn{4}{|l|}{$\begin{array}{l}\text { Table 2. Total numbers of correctly drawn figures on the } \\
\text { BVMT-R before and after treatment and at the first month }\end{array}$} \\
\hline & $\begin{array}{l}\text { MS group } \\
\text { (mean } \pm \text { SD) }\end{array}$ & $\begin{array}{l}\text { Control group } \\
\text { (mean } \pm \text { SD) }\end{array}$ & p \\
\hline Pretreatment & $20.59 \pm 8.41$ & $26.80 \pm 3.55$ & 0.001 \\
\hline Post-treatment & $25.59 \pm 8.97$ & - & - \\
\hline $\begin{array}{l}\text { First } \\
\text { month }\end{array}$ & $26.46 \pm 9.09$ & $27.96 \pm 2.92$ & 0.50 \\
\hline
\end{tabular}

BVMT-R: Brief visuospatial memory test-revised, MS: Multiple sclerosis, SD: Standard deviation

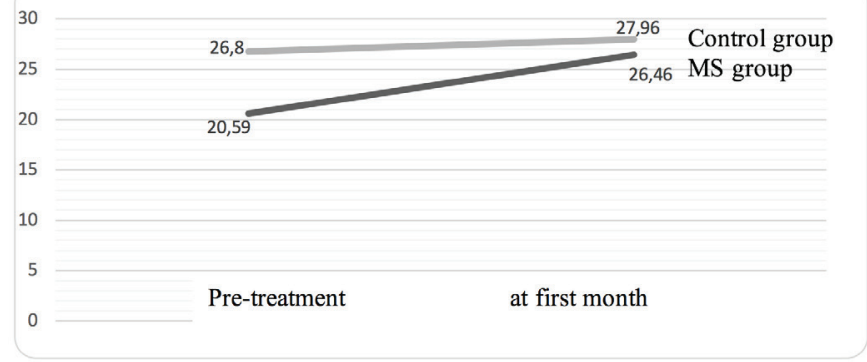

Figure 1. Changes in first-month BVMT-R scores compared to pre-treatment in the MS group

BVMT-R: Brief visuospatial memory test-revised, MS: Multiple sclerosis 


\begin{tabular}{|c|c|c|c|}
\hline & $\begin{array}{l}\text { MS group } \\
(\text { mean } \pm \text { SD) }\end{array}$ & $\begin{array}{l}\text { Control group } \\
\text { (mean } \pm \text { SD) }\end{array}$ & $\mathbf{p}$ \\
\hline Pretreatment & $42.04 \pm 11.73$ & $47.56 \pm 8.76$ & 0.055 \\
\hline Post-treatment & $45.40 \pm 16.32$ & - & - \\
\hline First month & $48.14 \pm 15.03$ & $49.24 \pm 9.06$ & 0.08 \\
\hline
\end{tabular}

SDMT: Symbol digit modalities test, MS: Multiple sclerosis, SD: Standard deviation

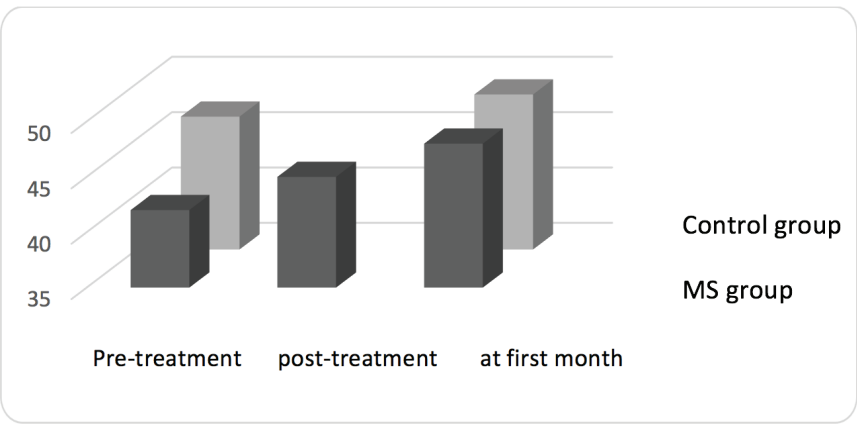

Figure 2. A comparison of the mean numbers of correctly drawn figures in 90 sec on the SDMT before and after treatment and at the first month in the MS and control groups

SDMT: Symbol digit modalities test, MS: Multiple sclerosis

of treatment, and at the first month after treatment (rho $=-0.165$, rho $=-0.264$, and rho $=-0.245$, respectively). BVMT-R and SDMT were found to be moderately correlated with EDSS at all three evaluations (Table 4). In the MS group, analysis of correlations among the cognitive tests revealed a good correlation between CVLT and SDMT before treatment and a moderate correlation between CVLT and BVMT-R and between BVMT-R and SDMT ( $r h o=0.519$, $r h o=0.407$, and $r h o=0.421$, respectively). At the end of treatment, a good correlation was noted between CVLT and BVMT-R, between CVLT and SDMT, and between BVMT-R and SDMT (rho=0.624, rho=0.551, and $\mathrm{rho}=0.494$, respectively). These good degrees of correlation also persisted at the first month (rho $=0.625$, rho $=0.476$, and $r h o=0.618$, respectively).

\begin{tabular}{|c|c|c|c|}
\hline & $\begin{array}{l}\text { Pretreatment } \\
\text { (rho value) }\end{array}$ & $\begin{array}{l}\text { Post-treatment } \\
\text { (rho value) }\end{array}$ & $\begin{array}{l}\text { First month } \\
\text { (rho value) }\end{array}$ \\
\hline $\begin{array}{l}\text { EDSS and } \\
\text { CVLT-II }\end{array}$ & -0.165 & -0.264 & -0.245 \\
\hline $\begin{array}{l}\text { EDSS and } \\
\text { BVMT-R }\end{array}$ & -0.319 & -0.307 & -0.285 \\
\hline $\begin{array}{l}\text { EDSS and } \\
\text { SDMT }\end{array}$ & -0.366 & -0.443 & -0.474 \\
\hline
\end{tabular}

SDMT: Symbol digit modalities test, BVMT-R: Brief visuospatial memory testrevised, CVLT: California verbal learning test II, MS: Multiple sclerosis

\section{Discussion}

Although individuals with MS present with highly diverse clinical manifestations, initial presentations are generally physical manifestations. Conditions that affect patients from the cognitive perspective are very rarely the reasons for the first presentation. Cognitive impairment is one of the factors, in addition to physical disability, that adversely and significantly affects the treatment process and quality of life of patients with MS. The identification and correction of these cognitive problems are also important in improving the quality of life by contributing to the therapeutic process.

The BICAMS battery consists of three tests evaluating different cognitive areas, namely, CVLT ॥, BVMT-R, and SDMT. CVLT ॥ assesses verbal learning, verbal memory, and immediate recall. BVMT-R evaluates visual-spatial learning and memory, and the SDMT assesses working memory, visual scanning, and rapid data processing (6). Baetge et al. (10) reported that the both of SDMT and BVMT-R were the most sensitive to cognitive impairment and the two test combinations showed the strongest agreement with the total battery. Studies recommending the use of the BICAMS battery are ongoing, although few studies have used the cognitive batteries in patients with MS in the attack period (11). In the present study, the cognitive functions of patients with MS were evaluated using the BICAMS battery before treatment, during treatment, and at the first month after treatment and were compared with those of healthy controls.

In the MS group, the number of words correctly recalled during the CVLT test increased significantly at the end of treatment and in the first month after treatment compared with that at baseline, although no significant difference was observed between the end-of-treatment and first-month values. This finding suggested that, in addition to improving physical symptoms, pulse therapy for an MS attack leads to improvement in cognitive state and that this improvement persists for an extended time after the attack period. A significant difference was also determined between the first-visit and first-month visit values in the control group. This can be attributed to the learning effect observed in the majority of cognitive tests. Comparison of the MS and control groups at the first visit and first-month visit revealed no significant difference between the two groups in either period. However, the significantly greater change at the first month compared with pretreatment in the MS group compared with that in the control group shows that the attack treatment in MS, rather than the learning effect, had affected the cognitive state.

In the MS group, the mean number of figures drawn correctly within $90 \mathrm{~s}$ on the SDMT test revealed significant improvement at the end of treatment and the first month compared with pretreatment values. While a significant difference was observed between pretreatment and first-month values in the MS group, a significant difference was also determined in the 
post-treatment and first-month values, suggesting that the improvement in the cognitive status commences in the early period and persists at the first month. Comparison of the MS group with the control group in terms of the mean numbers of correctly drawn figures within $90 \mathrm{~s}$ on the SDMT test at the first visit and at the first month revealed no significant difference. Similar to CVLT scores, the difference between pretreatment and first-month values was significantly greater in the MS group than in the control group. Moreover, similar to the CVLT, the SDMT can be used to evaluate the effectiveness of treatment. In agreement with other studies, this shows that the SDMT is a sensitive test of cognitive dysfunction $(12,13)$.

As regards the numbers of figures correctly drawn by the study group at each of the three trials on the BVMT-R, significant improvement was observed in the MS group after treatment and at the first-month compared with pretreatment levels. The significant difference with the control group before treatment suggests that the presence of cognitive impairment during an MS attack can be shown using this easily applied and understood test and that it may be more sensitive than the other two tests. As with SDMT and CVLT, the difference between pretreatment and fist-month values on the BVMT-R in the MS group was significantly higher than in the control group. This difference revealed by the three tests shows that cognitive areas such as information processing, verbal learning, and visuospatial perception are affected during MS attack and that some degrees of improvement occur with corticosteroid therapy. These findings are compatible with the results of a previous study that evaluates the use of the MSFC in the attack period of MS (14). Although the paced auditory serial addition test (PASAT) successfully shows the effectiveness of a treatment, these two similar studies show that the tests used in the present study can be employed instead of PASAT, which is relatively more difficult to apply and requires equipment. In addition, the use of SDMT has previously been recommended instead of PASAT on the MSFC, and our findings confirm the idea that SDMT can be effectively employed instead of PASAT in the attack period (15).

The present study also investigated whether EDSS, which is the most commonly used test for evaluating physical disability, but is not insufficient in the cognitive sphere, is associated with other cognitive tests. A moderate correlation was found between the EDSS and the BVMT-R and SDMT in the attack period, at the end of treatment, and at the first month after treatment, but the weak correlation observed with the CVLT II suggested that BVMT-R and SDMT are more sensitive in evaluating treatment response during attack and follow-up. A moderate or good correlation between the three cognitive tests in the attack period, after treatment, and at the first month after treatment is important in emphasizing the use of these scales in the attack period of MS in evaluating three different areas and providing detailed information about cognitive functions.
In 2018, Giedraitiene et al. (16) applied the BICAMS battery to patients with RRMS and stable MS and in healthy controls. Similar to the present study, the results of the SDMT, BVMT-R, and CVLT Il test were worse in the MS group than in the control group. Patients with RRMS registered lower scores than those with stable MS on the SDMT, while no significant difference was observed between the RRMS and stable MS groups on the other tests.

\section{Study Limitations}

In this study, the small number of patients was considered the most important limitation. Moreover, the addition of MRI parameters could help explain the paradox often mentioned in MS. The relatively low patient number in this study suggested that the difference in all cognitive tests might be low in the healthy control group. Further longitudinal studies evaluating cognitive functions in MS in both attack and remission periods will provide greater detail about such functions. When cognitive impairment is recognized as one of the most important parameters, showing the feasibility of the BICAMS battery in following response to attack treatment in patients with MS will encourage its wider application. All tests in the battery were easily understood by the patients, and no difficulties were encountered during application. Significant advantages also emerged, such as a total length of application as short as 15 min, and no neuropsychologist is required during application or interpretation. Comparison with the control group results shows the presence of cognitive impairment during the attack period. This study represents concrete evidence of an assumption that has long been held, but of which there has been little proof until now.

\section{Conclusion}

Cognitive involvement occurs in patients in the attack period of MS, and this is associated with widespread cerebral impairment in the attack period.

\section{Ethics}

Ethics Committee Approval: All procedures performed in studies involving human participants were in accordance with the ethical standards of the institutional and/or national research committee and with the 1964 Helsinki declaration and its later amendments or comparable ethical standards.

Informed Consent: Informed consent from all participiants was obtained for the study.

\section{Authorship Contributions}

Surgical and Medical Practices: O.O., B.P.C., S.O., Concept: O.O., B.P.C., S.O., Design: S.O., Data Collection or Processing: O.O., B.P.C., Analysis or Interpretation: B.P.C., S.O., Literature Search: O.O., B.P.C., S.O., Writing: O.O., B.P.C., S.O.

Conflict of Interest: No conflict of interest was declared by the authors. 
Financial Disclosure: The authors declared that this study received no financial support.

\section{References}

1. Chiaravalloti ND, DeLuca J. Cognitive impairment in multiple sclerosis. Lancet Neurol 2008;7:1139-1151.

2. Amato MP, Zipoli V, Portaccio E. Cognitive changes in multiple sclerosis. Expert Rev Neurother 2008;8:1585-1596.

3. Zipoli V, Goretti B, Hakiki B, Siracusa G, Sorbi S, Portaccio E, Amato MP. Cognitive impairment predicts conversion to multiple sclerosis in clinically isolated syndromes. Mult Scler 2010;16:62-67.

4. Takeda A. [Neuropsychological tests in multiple sclerosis]. Nihon Rinsho 2014;72:1989-1994. (Japanese)

5. Langdon DW, Amato MP, Boringa J, Brochet B, Foley F, Fredrikson S, Hämäläinen $\mathrm{P}$, Hartung HP, Krupp L, Penner IK, Reder AT, Benedict RH. Recommendations for a Brief International Cognitive Assessment for Multiple Sclerosis (BICAMS). Mult Scler 2012;18:891-898.

6. Benedict RH, Amato MP, Boringa J, Brochet B, Foley F, Fredrikson S, Hamalainen P, Hartung H, Krupp L, Penner I, Reder AT, Langdon D. Brief International Cognitive Assessment for MS (BICAMS): international standards for validation. BMC Neurol 2012;16:12-55.

7. Dusankova JB, KalincikT, Havrdova E, Benedict RH. Cross cultural validation of the Minimal Assessment of Cognitive Function in Multiple Sclerosis (MACFIMS) and the Brief International Cognitive Assessment for Multiple Sclerosis (BICAMS). Clin Neuropsychol 2012;26:1186-1200.

8. Penner IK, Filser M, Bätge SJ, Renner A, Ullrich S, Lassek C. Klinische Umsetzbarkeit der kognitiven Screeningbatterie BICAMS bei Patienten mit Multipler Sklerose: Ergebnisse der Machbarkeitsstudie in Deutschland [Clinical practicability of the cognitive screening battery BICAMS in patients with multiple sclerosis: results of the feasibility study in Germany]. Nervenarzt. 2021 Feb 17. (German) doi: 10.1007/s00115-021-01073-5. Epub ahead of print.

9. Thompson AJ, Banwell BL, Barkhof F, Carroll WM, Coetzee T, Comi G, Correale J, Fazekas F, Filippi M, Freedman MS, Fujihara K, Galetta SL, Hartung
HP, Kappos L, Lublin FD, Marrie RA, Miller AE, Miller DH, Montalban X, Mowry EM, Sorensen PS, Tintoré M, Traboulsee AL, Trojano M, Uitdehaag BMJ, Vukusic S, Waubant E, Weinshenker BG, Reingold SC, Cohen JA. Diagnosis of multiple sclerosis: 2017 revisions of the McDonald criteria. Lancet Neurol 2018:17:162-173.

10. Baetge SJ, Filser M, Renner A, Ullrich S, Lassek C, Penner IK. On the validity of single tests, two-test combinations and the full Brief International Cognitive Assessment for Multiple Sclerosis (BICAMS) in detecting patients with cognitive impairment. Mult Scler 2020;26:1919-1928.

11. Morrow SA, Jurgensen S, Forrestal F, Munchauer FE, Benedict RH. Effects of acute relapses on neuropsychological status in multiple sclerosis patients. J Neurol 2011;258:1603-1608. Erratum in: J Neurol 2011;258:1609.

12. Artemiadis A, Bakirtzis C, Chatzittofis A, Christodoulides C, Nikolaou G, Boziki MK, Grigoriadis N. Brief international cognitive assessment for multiple sclerosis (BICAMS) cut-off scores for detecting cognitive impairment in multiple sclerosis. Mult Scler Relat Disord 2021;49:102751. doi: 10.1016/j. msard.2021.102751.

13. Colato E, Stutters J, Tur C, Narayanan S, Arnold DL, Gandini WheelerKingshott CAM, Barkhof F, Ciccarelli O, Chard DT, Eshaghi A. Predicting disability progression and cognitive worsening in multiple sclerosis using patterns of grey matter volumes. J Neurol Neurosurg Psychiatry 2021:jnnp2020-325610. doi: 10.1136/jnnp-2020-325610. (Epub ahead of print)

14. Ozakbas S, Cagiran I, Ormeci B, Idiman E. Correlations between multiple sclerosis functional composite, expanded disability status scale and healthrelated quality of life during and after treatment of relapses in patients with multiple sclerosis. J Neurol Sci 2004;218:3-7.

15. Strober L, DeLuca J, Benedict RH, Jacobs A, Cohen JA, Chiaravalloti N, Hudson LD, Rudick RA, LaRocca NG; Multiple Sclerosis Outcome Assessments Consortium (MSOAC). Symbol Digit Modalities Test: A valid clinical trial endpoint for measuring cognition in multiple sclerosis. Mult Scler 2019;25:1781-1790.

16. Giedraitiene N, Kaubrys G, Kizlaitiene R. Cognition during and after multiple sclerosis relapse as assessed with the brief international cognitive assessment for multiple sclerosis. Sci Rep 2018;8:8169. 